\title{
Do User-Defined Gestures for Flatscreens Generalize to Interactive Spherical Displays for Adults and Children?
}

\author{
Nikita Soni ${ }^{1}$, Schuyler Gleaves ${ }^{1}$, Hannah Neff ${ }^{4}$, Sarah Morrison-Smith ${ }^{1}$, Shaghayegh Esmaeili ${ }^{1}$, Ian \\ Mayne $^{6 \dagger}$, Sayli Bapat ${ }^{5 \dagger}$, Carrie Schuman ${ }^{3}$, Kathryn A. Stofer ${ }^{2}$, Lisa Anthony ${ }^{1}$ \\ ${ }^{1}$ Dept. of CISE, ${ }^{2}$ Dept. of Agricultural Education \& Communication, ${ }^{3}$ School of Natural Resources and Environment, \\ ${ }^{4}$ Dept. of Sociology, University of Florida, Gainesville, FL, USA. ${ }^{5}$ Maharashtra Institute of Technology Pune, India, ${ }^{6}$ Elon \\ University, Elon, NC, USA. Contact authors: nsoni2@ufl.edu, stofer@ufl.edu, lanthony@cise.ufl.edu \\ $\dagger$ Work conducted while this author was a summer intern at the University of Florida
}

\begin{abstract}
Interactive spherical displays offer unique opportunities for engagement in public spaces. Research on flatscreen tabletop displays has mapped the gesture design space and compared gestures created by adults and children. However, it is not clear if the findings from these prior studies can be directly applied to spherical displays. To investigate this question, we conducted a user-defined gestures study to understand the gesture preferences of adults and children (ages 7 to 11) for spherical displays. We compare the physical characteristics of the gestures performed on the spherical display to gestures on tabletop displays from prior work. We found that the spherical form factor influenced users' gesture design decisions. For example, users were more likely to perform multi-finger or whole-handed gestures on the sphere than in prior work on tabletop displays. Our findings will inform the design of interactive applications for spherical displays.
\end{abstract}

\section{CCS CONCEPTS}

- Human-centered computing Touch screens

\section{KEYWORDS}

interactive spherical displays, user-defined gestures, touchscreen displays, adults, children

\section{ACM Reference format:}

Nikita Soni, Schuyler Gleaves, Hannah Neff, Sarah MorrisonSmith, Shaghayegh Esmaeili, Ian Mayne, Sayli Bapat, Carrie Schuman, Kathryn A. Stofer, Lisa Anthony. 2019. Do User-Defined Gestures for Flatscreens Generalize to Interactive Spherical Displays for Adults and Children?. In Proceedings of $A C M$ Pervasive Displays conference (PerDis'19). ACM, New York, NY, USA, 7 pages. https://doi.org/10.1145/3321335.3324944

Permission to make digital or hard copies of part or all of this work for personal or classroom use is granted without fee provided that copies are not made or distributed for profit or commercial advantage and that copies bear this notice and the full citation on the first page. Copyrights for components of this work owned by others than ACM must be honored. Abstracting with credit is permitted. To copy otherwise, or republish, to post on servers or to redistribute to lists, requires prior specific permission and/or a fee. Request permissions from permissions@acm.org.

PerDis '19, June 12-14, 2019, Palermo, Italy

(C) 2019 Association for Computing Machinery

ACM ISBN 978-1-4503-6751-6/19/06..\$15.00

https://doi.org/10.1145/3321335.3324941

\section{INTRODUCTION}

Beyond flatscreen displays such as multi-touch tabletops, there is a growing interest in designing educational and entertainment applications for interactive spherical displays, in public settings $[10,29,30]$. Spherical displays have been deployed in museums as a teaching support for, e.g., mathematics or geography [13], but they either support no interactivity, or interaction only via an external touchscreen [29,30]. These configurations present challenges in using spherical displays as an educational platform. For example, using a separate interface to manipulate content displayed on the sphere can increase the cognitive demand placed on learners trying to operate the interface and view the content on two separate screens [14]. In 2008, Benko et al. [4] analyzed how interaction techniques such as drag and flick could be implemented on a prototype touch-driven spherical display. Only recently have spherical displays become commercially available that can support such multi-touch gesture interactivity [31,32]. Although significant progress has been made in terms of hardware and software capabilities [9,31], little work has been done to identify specific touch interaction that would be intuitive to all users and age groups. Touch interaction techniques for spherical displays that have been developed [4,5] have primarily been designer-driven. However, for real-world use, designerdefined interactions are less discoverable and natural for users on new platforms than gestures designed by end users [16].

Prior research on flatscreen tabletop displays has mapped the gesture design space [28] and compared gestures created by adults and children [20]. However, it is not clear if the findings from these prior studies can be directly applied to interactions with spherical displays for two reasons. Firstly, spherical displays, which show the entire digital world in a continuous fashion, are metaphorically different than flatscreen displays, which act as a viewport into the larger digital world [4]. This metaphorical difference could afford different interaction styles for spherical displays than those that are commonly seen with flatscreen displays. Secondly, unlike flatscreen displays, a spherical display combines the properties of two form factors. The top portion of the display is a shared, almost horizontal surface (e.g., tabletops), while the sides of the sphere are more like vertical displays (e.g., smart walls) [4]. It has been shown that these form factors afford different interaction styles [3]. Thus, if we could characterize what differences exist in users' gesture preferences between 
spherical and flatscreen displays, we could provide clearer interaction design recommendations for spherical displays.

The primary goal of this paper is to investigate how adults and children (ages 7 to 11, ages typically targeted by public learning exhibits [1]) interact with large spherical displays. To help us understand users' gesture preferences and how the form factor of the display might influence the types of gestures users would find more intuitive on the spherical display as opposed to flatscreen tabletop displays, we conducted a user study by adapting the userdefined gestures methodology from Wobbrock et al. [28]. It is important to study both children and adults because results from touchscreen studies with adults have been shown to differ for children, who have different motor and cognitive capabilities [2].

We found that the spherical form factor influenced users' gesture design decisions. Users were more likely to perform multifinger or whole-handed gestures on the sphere than in prior work on tabletop displays. We also observed children were more likely than adults to use their left and right hands interchangeably and to try gestures with dynamic vs. static hand poses. The contributions of this paper are: (1) classification of user-defined gestures for spherical displays, based on a gesture taxonomy grounded in prior work and adapted for this context; (2) comparison of user-defined spherical display gestures to those identified in prior work for flatscreen tabletops and walls; (3) characterization of similarities and differences in the gestures that children propose versus those of adults, in terms of the gesture taxonomy. These findings will aid designers in better supporting touch gestures for spherical interfaces for users of all age groups.

\section{RELATED WORK}

Our review focuses on: (1) interactions for spherical displays; and (2) gesture elicitation studies (user-defined gestures method).

\subsection{Interactions for Spherical Displays}

Prior work has explored the use of non-interactive spherical displays for learning. Hsi and Eisenberg [13] used "Science on a Sphere" (SoS) for teaching geometry and computational thinking to children in science centers. They found that using a spherical interface kept children engaged and helped them learn geometry concepts, though they did not compare to flatscreens directly.

Benko et al. [4] developed the first interactive spherical display in 2008, and presented a set of designer-defined interactions such as dragging and scaling of objects. The authors also implemented touch interaction techniques such as flicking and send-to-dark-side to facilitate collaboration around spherical displays. However, these techniques were not evaluated with end-users to understand their gesture preferences. Bolton et al. [5] explored competitive and cooperative tasks on spherical displays in a lab-based study, implementing multiple software-based "peeking" techniques to allow users to see the other parts of the display. Since interactive spherical displays have only recently become commercially available [31,32], most of this prior work has established the parameters of interaction in lab-based studies. A notable exception is an in-the-wild study conducted by Williamson et al. [24] that examined how supporting different types of interactions affected dwell times at the spherical display: offering more interactive options increased dwell times as users explored more of the features. While prior work has studied interaction around spherical displays, most of the interactions are designer-defined, which are more conceptually complex and less preferred than those designed by end-users [16]. Hence, more work is needed to understand users' gesture preferences for spherical displays.

\subsection{Gesture Elicitation Studies}

Several studies have used the user-defined gestures method, introduced by Wobbrock et al. [28], to elicit gestures from users (hence the other common name: gesture elicitation study) to maximize gesture guessability [27] while disregarding technical concerns. In this approach, users are shown an interface effect (known as a referent) and are asked to perform gestures they think are appropriate to cause the effect. The elicited gestures can be classified using a gesture taxonomy [28] to understand their structure (e.g., hand pose, number of fingers); this analysis provides insight into users' preferences with respect to gesture interactions in specific contexts or with specific form factors.

Prior work comparing user-defined and designer-defined gesture sets found that users prefer user-defined gestures, because designer-defined gestures are often more complex and based on nuanced technical knowledge of the system [16]. Several studies have elicited gestures for touchscreen platforms, including large public displays [20,26,28]. Rust et al. [20] replicated the original Wobbrock et al. [28] tabletop gesture elicitation study with children and adults to understand children's gesture preferences for interaction with multi-touch tabletops. They found that the gestures created by both age groups exhibited "legacy bias" [17], resulting in gestures influenced by prior touchscreen experience. Wittorf and Jakobsen [26] conducted a gesture elicitation study with adults for understanding wall-display interactions. They found that users' gestures were largely influenced by prior touchscreen experience, but tended to be larger variations in comparison to prior work with small touchscreens. For example, for zooming an object, most participants suggested two-handed spreads, and only a few participants suggested finger spreads.

While these previous studies offer a solid foundation for understanding users' gesture preferences for flatscreen displays, these results may not generalize to spherical displays. In addition, the gesture elicitation methodology has only been employed with children to a limited extent $[8,20]$. Children have less fine motor control and different cognitive capabilities than adults [2,19]. These developmental capabilities of children contribute to key differences in how they interact with touchscreen interfaces. Thus, we used the gesture elicitation study method to understand both adults' and children's gesture preferences for spherical displays, and to compare the gestures they proposed to gestures previously proposed for multi-touch tabletops [28]. These insights will be enable design of intuitive gestures for spherical displays.

\section{SPHERICAL DISPLAY ELICITATION STUDY}

We elicited touch gestures using an adaptation of the gesture elicitation approach [28]. Below we describe our methodology. 




Figure 1: Participant proposing gesture for Copy referent, originally used by Rust et al. [20].

\subsection{Task Selection}

Our study included 17 referents divided into two higher-level categories: (1) traditional touchscreen referents, and (2) spherespecific referents. For \#1, to enable direct comparison with the gesture elicitation studies conducted with tabletop displays, we included many of the same referents that were included by Wobbrock et al. [28] and Rust et al. [20]; these referents are marked with (t) in the list below. Our referent set contained nine traditional touchscreen referents, including one practice referent. For \#2, we added eight sphere-specific referents, marked $\mathbf{s}$ in the list below, based on prior work on spherical displays $[4,13,24,25]$. Sphere-specific referents only make sense on the sphere form factor, such as rotating the sphere and sending an object to the other side of the sphere. Below is the list of all 17 referents:

- Practice: Bigger $(\mathrm{t})$

- Group 1: Turn Sphere in X (s), Turn Sphere in Y (s), Smaller (t), Flick (s), Stop Rotation (s), Move an Object (t)

- Group 2: Send to the Other Side (s), Next (t), Reset Sphere Rotation (s), Undo (t), Copy (t)

- Group 3: Remove (t), Pick One (t), Pick Many (t), Local Rotation in X (s), Local Rotation in Y (s)

\subsection{Participants}

A total of 33 participants (13 adults and 20 children) participated in our study. Five of the children were pilot study participants to help us improve our study protocol for this population. After the pilots, we introduced a think-aloud practice task, since children tended to not talk out loud about why they proposed a gesture. Two other child participants opted not to complete the study. Therefore, data from 26 participants (13 adults and 13 children) was considered for our final analysis. Adults (9F, 4M) ranged in age from 19 to 52 (M: 31.58, SD: 10.03), while children (5F, 8M) were recruited to be 7 to 11 years old (M: 9.20, SD: 1.44$)$. We recruited participants via an email sent to a faculty list and through flyers distributed at a local museum and library. The sessions were conducted in a private room in a research lab or public library. Most children and adults (85\% each) rated their general touchscreen familiarity as "average" or "expert". In addition, most had interacted with touchscreen exhibits in science museums (children: $77 \%$ and adults: 100\%). Each of the participants was given $\$ 10$ and three small prizes for participating.

\subsection{Study Methodology}

During our study, participants proposed gestures for 17 total referents, including one practice referent. The 16 main referents were divided into three groups, as shown in the bulleted list, providing a mix of sphere-specific and traditional touchscreen referents within each group. The order of the groups was systematically counterbalanced across participants using a Latin Square; the order of referents within each group was kept constant. Informed by earlier work on techniques to reduce legacy bias in elicitation studies [17], we asked participants to propose three gestures for each referent (i.e., the production technique), with the aim of promoting diversity in the gestures produced. We asked participants to come up with two one-handed gestures and one two-handed gesture. To maximize children's completion rates in this repetitive study, we added gamification elements, namely, a score that increased for each gesture, based on prior work [6].

Each participant was read the informed consent or assent before deciding to participate. After the consent process, we instructed participants in how to use the think-aloud process [11], and did a practice think-aloud with them while solving a twocolumn addition problem. To overcome the novelty effect of the spherical display [20], we added a 5-minute play period with a fireworks application. The experimenter then walked the participant through the practice referent and the gesture rating questions, after which the 16 main referents were shown. For each referent, the experimenter read a brief description, then played a video demonstrating the referent. The first frame of the video was shown again, and the experimenter asked the participant to think aloud and propose a gesture that they thought would cause the demonstrated effect (Figure 1). Since participants were asked to propose three gestures per referent, the video was played three times for each referent. We provide the videos of all the referents seen by the participants as a supplemental video with this paper. After participants proposed three gestures per referent, they were asked to rate each gesture for goodness of fit ("The gesture I picked is a good match for its intended use.") and ease of use ("The gesture I picked is easy to perform.") [28]. Participants filled out a demographics questionnaire at the end of the study. Our protocol was approved by our Institutional Review Board.

The study was conducted using a Puffersphere $600 \mathrm{M}$ [31], a 24" diameter commercially available spherical display with a resolution of up to $1600 \times 1600$ pixels (34 ppi). The display is 1475 $\mathrm{mm}$ tall (58 in) and uses interior cameras to capture touches. We wrote a C\# application to present referent videos to the participants. All the sessions were recorded with two video cameras recording over both shoulders of the participant. Participants' touches were also logged by the application running on the sphere, though analysis of the touch logs is not presented in this paper. This paper focuses on characterizing the gesture design space based on user behavior on spherical displays, and comparing our results to prior work with flatscreen displays.

\subsection{Data Analysis Procedure}

With 26 participants, 16 referents, and 3 gestures per referent, a total of 1248 gestures were made (excluding the practice referent). 
(a)

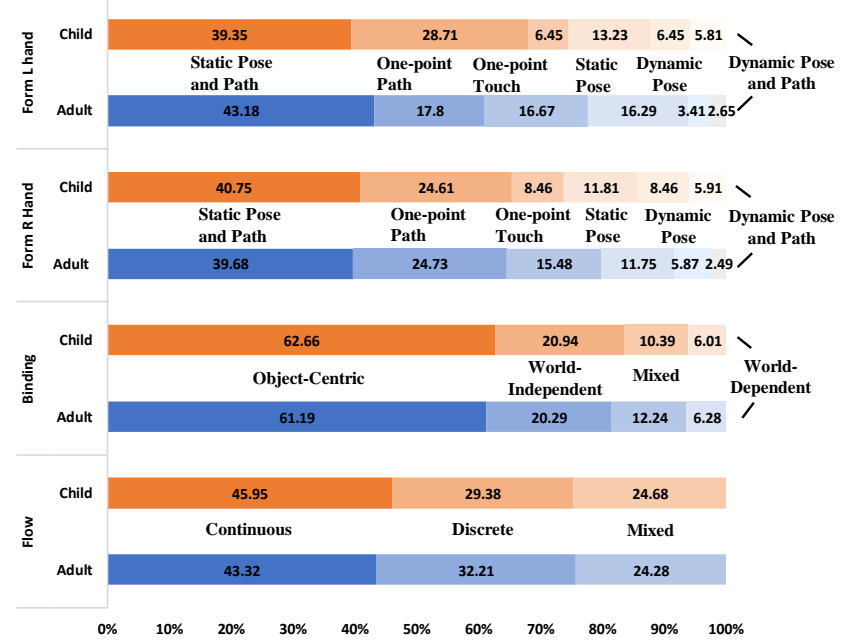

(b)

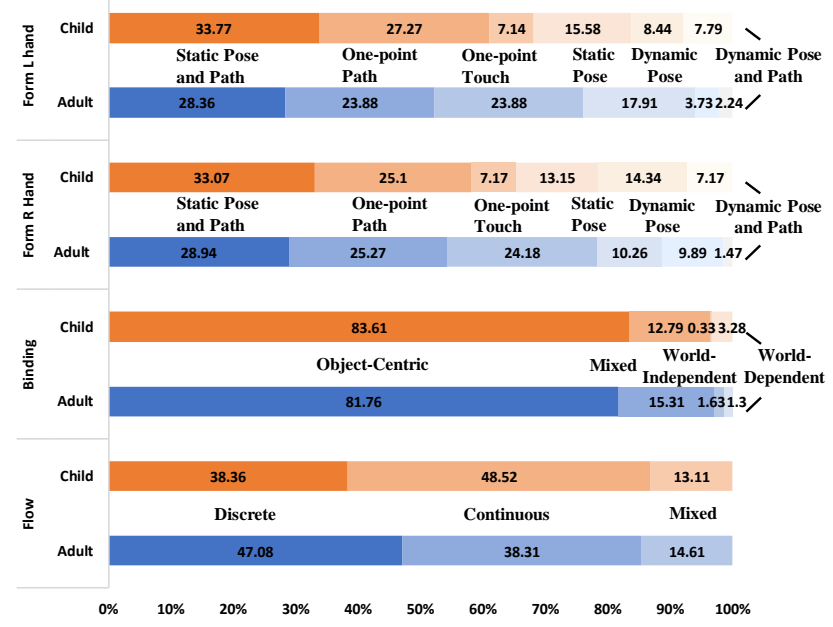

Figure 2: Results of gesture classification for our full set of referents in (a) and only the traditional touchscreen referents in (b). Total number of gestures ( $N$ ), in (a) for Form $L(N=264$ (adult) and $N=310$ (child)), Form $R(N=564$ (adult), $N=509$ (child)) and in (b) for Form $L$ ( $N=134$ (adult) and $\mathrm{N}=154$ (child)), Form $\mathrm{R}(\mathrm{N}=275$ (adult) $\mathrm{N}=250$ (child)). For Binding and Flow, $\mathrm{N}=622$ (adult), $\mathrm{N}=616$ (child) in (a) and $\mathrm{N}=308$ (adult), $\mathrm{N}=305$ (child), in (b). For bimanual gestures, Form $\mathrm{R}$ and Form $\mathrm{L}$ were applied separately to each hand.

Of these, 8 gestures by children and 2 gestures by adults were discarded due to inability of the participant to come up with a gesture for that referent. We analyzed a total of 1238 gestures (622 adult and 616 child gestures), corresponding to 47 or 48 gestures per participant. To facilitate comparison between user-defined gestures for spherical and flatscreen displays, we coded the elicited gestures based on four dimensions (form $L / R$ hand, binding, flow) from Wobbrock et al.'s taxonomy of the gesture design space for tabletop displays [28]. Details on each dimension and its relevant categories are provided with our findings. Due to space constraints, we do not present the results of the think-aloud analysis here, but leave that for a future paper.

We qualitatively coded our 1238 gestures in a three-phase coding process. In the first two phases, four researchers coded the same set of two randomly chosen participants (one adult and one child) along the dimensions of Wobbrock et al.'s taxonomy, and a discussion of agreements and disagreements led to refinement of the coding dimensions. In the third phase, the four researchers independently coded gestures from 6 or 7 participants each. Each pair of researchers additionally coded a $10 \%$ random sample from each other's data to compare interrater reliability. The average Cohen's kappa across all dimensions and coders was 0.69 (min: 0.56 , max: 0.83 , SD: 0.12 ). This value corresponds to "substantial" agreement [15]; however, in our data, Cohen's kappa overpenalizes disagreements because many gestures are similar in most dimensions [12]. Overall percent agreement without accounting for chance across all dimensions and coder pairs was 81\% (min: 74\%, $\max : 89 \%$, SD: 7\%). Before final analysis, we discussed disagreements to ensure that they were due to reasonable subjective judgements and not conceptual misunderstanding. Our first analysis goal was to understand the characteristics of the participants' gestures for spherical displays, so we analyzed all the gestures for our full set of 16 referents, i.e., both sphere-specific and traditional touchscreen referents (Figure 2-a), which included 1238 gestures: 622 for adults and 616 for children. Our second goal was to understand how user-defined spherical display gestures differ from those identified for tabletops by Wobbrock et al. [28], so we directly compared the gestures performed on just the eight traditional touchscreen referents in our study to the tabletop display gestures in Wobbrock et al.'s study. This analysis included 613 gestures: 308 for adults and 305 for children (Figure 2-b). We also analyzed adults' and children's gestures separately to understand differences between them.

\section{FINDINGS FROM GESTURE CODING}

\subsection{Form}

The form dimension's scope as defined by Wobbrock et al. [28] covers one hand, and in the case of bimanual gestures, we applied it separately for each hand. As in Wobbrock et al.'s taxonomy, the form dimension was divided into multiple categories. A gesture is considered one-point [touch or path] if the user touches the sphere with only one finger (e.g., one-point touch: one-finger tap and onepoint path: one-finger drag). A gesture is considered static pose when the hand pose is held constant at one location (e.g., touching the sphere with an open palm), and static pose and path when the hand pose is held constant as the hand moves (e.g., two-finger swipe). A gesture's form is dynamic pose when the hand pose changes at the same location (e.g., pinching at one spot using all five fingers) and dynamic pose and path when the hand pose changes as the hand moves (e.g., pinching while dragging).

In Wobbrock et al.'s paper, they saw that within the form dimension the most common gestures were one-point path (45.0\%). In contrast, in our full referent set, the most common right-handed gestures for both children and adults were static pose and path ( $40.75 \%$ and $39.68 \%$, respectively), and the second most frequent gesture type was one-point path (24.61\% and 24.73\%) (Figure 2-a). An example of a static pose and path gesture proposed by multiple 
participants includes using both hands (all fingers) to swipe upwards for the referent turn sphere in $X$. Even when we just compare the traditional touchscreen referents with Wobbrock et al.'s results (Figure 2-b), we still see a larger proportion of static pose and static pose and path gestures in our study than in theirs: static pose (10.26\% vs. $3.7 \%$, respectively) and static pose and path ( $28.94 \%$ vs. $14.0 \%)$. Across both studies, the occurrence of dynamic pose and dynamic pose and path gestures was low (under 10\%). An example of a static pose and path gesture proposed for a traditional touchscreen referent is using one hand (all fingers) to drag an object towards the top of the sphere and then bringing in the next object from the right side of the sphere for the referent next.

Comparing children's gestures to adults' gestures for the form dimension reveals some differences. For our full referent set, we found that children proposed a lower proportion than adults of one-point touch gestures ( $8.46 \%$ vs. $15.48 \%$, respectively) and a higher proportion than adults of dynamic pose ( $8.46 \%$ vs. $5.87 \%$ ) and dynamic pose and path (5.91\% vs. $2.49 \%$ ) gestures (Figure 2-a). Even when we just compare on the traditional touchscreen referents, children also proposed a higher proportion than adults of dynamic pose and path (7.17\% vs. $1.47 \%$ ) gestures (Figure 2-b). An example of this type of gesture proposed by a child includes pinching over an object using all five fingers while swiping to undo an action. Although most of the children (85\%) and adults (92\%) in our study were right-handed, children tended to use their left hand more frequently when gesturing $(50.3 \%, \mathrm{~N}=616)$ than adults $(42.4 \%, \mathrm{~N}=622)$. In general, both children and adults more often proposed gestures that involved usage of multiple fingers on spherical displays as compared to tabletop displays, but children were more likely than adults to use their left and right hands interchangeably and try gestures with dynamic hand poses.

\subsection{Binding}

As in Wobbrock et al.'s [28] paper, we coded gestures in the binding dimension based on the relative position where they were performed on the sphere. Object-centric covers gestures that require information about the object being manipulated, e.g., pinching to make an object smaller. Gestures such as dragging an object towards the bottom of the display to remove it are worlddependent gestures, as they depend on the location of interaction with respect to the world. World-independent gestures occurred anywhere on the display, regardless of the relative position to the world, e.g., touching anywhere on the sphere interface to stop rotation. Gestures coded as mixed-dependent were usually worldindependent in one aspect and world-dependent or object-centric in another aspect. For example, we coded a gesture for moving an object in which selection of the object to be moved is object-centric and object placement is world-independent as mixed-dependent.

In Wobbrock et al.'s paper, they saw that a majority of gestures were object-centric $(70.2 \%)$ and a low proportion of gestures were world-independent $(8.9 \%)$ or mixed $(2.7 \%)$. Similarly, in our full referent set, we found that the majority of gestures were objectcentric (61.19\%) (Figure 2-a). In contrast, we did see a larger proportion of world-independent gestures in our full set of referents (20.29\%) than Wobbrock et al.'s paper (8.9\%). An example of a world-independent gesture includes resetting sphere rotation using one hand (all fingers, open palm) and tracing a circle anywhere on the interface, regardless of the relative position to the world. However, when we just looked at the traditional touchscreen referents, we saw a very low proportion of world-independent gestures for both children $(0.33 \%)$ and adults (1.63\%) (Figure 2-b). Digging into this further, for the eight spherespecific referents alone, we found higher proportions of worldindependent gestures than for traditional touchscreen referents for both adults ( $38.54 \%$ vs. $1.62 \%$, respectively) and children $(41.16 \%$ vs. $0.33 \%$ ). Also, in contrast to Wobbrock et al.'s observation $(2.7 \%)$, we saw a larger proportion of mixed gestures in our study $(12.24 \%)$. Generally, participants proposed world-independent gestures for sphere-specific referents such as resetting sphere rotation, but for traditional touchscreen referents, participants more often proposed object-centric or world-dependent gestures.

\subsection{Flow}

We adapted the flow dimension from Wobbrock et al.'s paper [28] for our study. A gesture's flow is discrete if the effect of a gesture takes place only after the gesture is completed (e.g., an index finger should trace a complete $\mathrm{R}$ symbol to reset sphere rotation). It is continuous if the effect of the gesture should take place along with the gesture being performed (e.g., pinching gesture). We extended Wobbrock et al.'s categories for the flow dimension to include partially continuous, or mixed, gestures. Although all nondiscrete gestures can be thought of as continuous, we saw participants specifically proposing compound gestures (e.g., double tap and drag) or gestures during which participants thought that the interface would keep responding (or changing) during the gesture as well as after the gesture is done. These seemed important to capture separately. An example is the flick gesture, in which the object travels along with the user's swipe gesture and continues once the swipe is complete. While performing this gesture, the participant assumed that the object would continue to travel in the same direction even after the participant was no longer guiding or touching the object.

In Wobbrock et al.'s paper, within the flow dimension, a majority of gestures were discrete (53.5\%). In contrast, for our full set of referents, the most common gestures were continuous for both children (45.95\%) and adults (43.32\%) (Figure 2-a). However, when we just looked at the traditional touchscreen referents, we observed a larger proportion of discrete gestures (47.08\%) for adults than in our full set of referents (Figure 2-b). In addition, we observed a higher proportion of mixed gestures for our full set of referents than traditional touchscreen referents for both children ( $24.68 \%$ vs. $13.11 \%$, respectively) and adults ( $24.28 \%$ vs. $14.61 \%)$.

We also saw differences when comparing children's gestures to adults' gestures. Digging into this further, for the eight spherespecific referents alone, we found that, in general, adults proposed more continuous gestures than children for sphere-specific referents ( $48.41 \%$ vs. $43.41 \%$, respectively) and more discrete gestures for traditional touchscreen referents ( $47.08 \%$ vs. $38.31 \%$ ). In contrast, children tended to propose more continuous than discrete gestures for both sphere-specific (43.41\% vs. $20.58 \%)$ and traditional touchscreen referents $(48.52 \%$ vs. $38.62 \%)$. Overall, participants were more likely to propose continuous gestures. 
Specifically, we observed a higher proportion of continuous gestures for sphere-specific referents and a higher proportion of discrete gestures for traditional touchscreen referents for adults; however, children tended to use continuous gestures in general.

\section{DISCUSSION}

The main goal of our study was to understand how users' gestures differ from the flatscreen context to the spherical context. Next, we discuss elements of the spherical display that seemed to influence how users proposed gestures, and we compare userdefined spherical display gestures to those identified in prior work on other platforms including touch walls.

Influence of the Vertical Nature of the Sphere (Form Dimension). Anthony et al. [3] and Rust et al. [20] studied gestural interactions for tabletop displays and found that most gestures people attempted were standard touchscreen gestures (e.g., tap, drag) that did not involve usage of multiple fingers. Anthony et al. [3] also studied gestural interactions for vertical wall displays and saw that users were more likely to perform gestures that involved usage of multiple fingers on touch walls than on tabletop displays. They note that this difference might be due to different ergonomics affordances between the two form factors. Similarly, Wittrof and Jakobsen [26] in their work on interactive wall displays showed a majority of the gestures people performed included multiple fingers or the whole hand. Consistent with this prior work, for spherical displays our participants proposed more standard touchscreen gestures that involved usage of more than one finger or the whole hand (i.e., static pose and path gestures), commenting on the vertical nature of the display, as in the following think-aloud utterances: "...on a sphere it would just make more sense because of it being a sphere instead of just tapping it ... because [the phone] is flat but on a sphere, you should use all your fingers." [child-pick one] and "It feels like if this was a picture on a wall, then I am trying to adjust it. I would take the corners and rotate them." [adult-reset sphere rotation]. The spherical display is perhaps more analogous to a vertical wall display, since the sides of the sphere are oriented vertically in relation to the user [4]. This pattern of using gestures that involve multiple fingers on the spherical display as opposed to horizontal tabletops could be due to similarity in ergonomic affordances between touch walls and spherical displays. Since our users proposed gestures that involve usage of both one and multiple fingers for the same referents, interactions for spherical displays should support both single-touch and larger variants of gestures, such as one-and two-handed gestures for the same command, to make cross-platform gestural interaction more intuitive for users.

Preference for World-Independent Gestures on the Sphere (Binding Dimension). Both Wobbrock et al. [28] and Wittrof and Jakobsen [26] in their work on tabletop and interactive wall displays, respectively, observed a low prevalence of world-independent gestures as compared to world-dependent and object-centric gestures. Similarly, in our study users were more likely to propose object-centric or world-dependent gestures for traditional touchscreen referents. In contrast, for spherespecific referents, a majority of gestures our users proposed were world-independent. Users expected the sphere to differentiate if the gesture should be applied to an object or the whole sphere, as in this utterance: "I want to make it more direct so that I do not select something, so I will use multiple fingers to stop it." [adult-stop rotation]. To support this expectation, the sphere should be able to recognize and differentiate between these two cases. To ensure that the gesture is applied to an object, some users double tapped an object first to select it and then gestured. Future work should examine differences in users' mental models while they perform object-centric and world-independent gestures to understand cues they may use in their gestures to signify the difference.

Differences between Children and Adults (Form and Flow Dimensions). We saw some differences when comparing children's gestures to adults' gestures across multiple dimensions. Both Anthony et al. [3] and Rust et al. [20] in their work with flat touchscreen displays with children and adults showed that children tend to try unique (i.e., nonstandard) gestures more frequently than adults. Our observations confirm this behavior is even more prevalent for spherical displays. For example, one child participant reset the sphere rotation by dragging one hand (four fingers) in a circular motion: "It is like the spiral except it is in one big circle. I was thinking the sphere would rewind." [child-reset sphere rotation]. We saw that children were more likely than adults to use their left and right hands interchangeably and propose gestures that involved dynamic hand poses during their interaction with the sphere, for example, by pinching over an object using all five fingers while swiping it to undo an action. We also observed children proposing more continuous gestures that require ongoing recognition as the gesture is being performed and fewer discrete one-touch gestures, than did adults. Children's inclination towards trying unique gestures is likely due in part to their tendency to explore and their curiosity about objects around them [19,21]. Touchscreens allow children to directly control interface objects, which enables them to interact with objects in a way that would not be possible with a traditional mouse [18]. Thus, to design interfaces to support children's cognitive abilities and inventiveness, novel form factors like spherical displays must respond meaningfully to unanticipated gestures.

\section{LIMITATIONS AND FUTURE WORK}

Our findings point to how both the spherical form factor and developmental differences between children and adults influence users' gesture preferences. Since Wobbrock et al.'s [28] study was conducted with people with comparatively less touchscreen experience than those in our study, prior touchscreen experience is a relevant factor in our comparison between platforms. Also, our study used a commercially available spherical display that was 24 " in diameter; future work should study whether the size of the sphere would make a difference in how users propose gestures. Future work could also compare our results to other user-defined gestures beyond touchscreens [7,8,22,23]. Our paper reports physical characteristics of gestures people find intuitive to inform the design of interactions for spherical displays, which is important as such displays become more widespread in public spaces. For future work, we plan to conduct more in-depth 
analysis on data from our study about users' mental models and to propose a user-defined gesture set for spherical displays.

\section{ACKNOWLEDGEMENTS}

This work is partially supported by National Science Foundation Grant Awards \#DRL-1612485 and \#IIS-1552598. Any opinions, findings, and conclusions or recommendations expressed in this paper are those of the authors and do not necessarily reflect these agencies' views. The authors thank Wobbrock et al. [28] and Rust et al. [20] for providing access to their original referent videos; Dr. Jaime Ruiz, Isaac Wang, Aishat Aloba, Julia Woodward, and Jesse Smith for assistance with this work; Pufferfish Ltd. for providing technical support; and the Florida Museum of Natural History, PK Yonge Developmental Research School, and the Alachua County Library System for allowing us to recruit participants from their visitors and students.

\section{REFERENCES}

[1] Amanda Harris, Jochen Rick, Victoria Bonnett, Nicola Yuill, Rowanne Fleck, Paul Marshall, and Yvonne Rogers. 2009. Around the Table: Are Multiple-Touch Surfaces better than Single-Touch for Children's Collaborative Interactions? In Proceedings of the Conference on Computer Supported Collaborative Learning (CSCL'09), 335-344.

[2] Lisa Anthony, Quincy Brown, Jaye Nias, Berthel Tate, and Shreya Mohan. 2012 Interaction and Recognition Challenges in Interpreting Children's Touch and Gesture Input on Mobile devices. In Proceedings of the Conference on Interactive Tabletops and Surfaces (ITS'12), 225-234.

[3] Lisa Anthony, Kathryn A. Stofer, Annie Luc, and Jacob O. Wobbrock. 2016. Gestures by Children and Adults on Touch Tables and Touch Walls in a Public Science Center. In Proceedings of the International Conference on Interaction Design and Children (IDC'16), 344-355.

[4] Hrvoje Benko, Andrew D. Wilson, and Ravin Balakrishnan. 2008. Sphere: Multitouch Interactions on a Spherical Display. In Proceedings of the ACM Symposium on User Interface Software and Technology, 77-86.

[5] John Bolton, Kibum Kim, and Roel Vertegaal. 2012. A Comparison of Competitive and Cooperative Task Performance using Spherical and Flat Displays. In Proceedings of the Conference on Computer Supported Cooperative Work (CSCW'12), 529-538.

[6] Robin Brewer, Lisa Anthony, Quincy Brown, Germaine Irwin, Jaye Nias, and Berthel Tate. 2013. Using Gamification to Motivate Children to Complete Empirical Studies in Lab Environments. In Proceedings of the Conference on Interaction Design and Children (IDC'13), 388-391.

[7] Edwin Chan, Teddy Seyed, Wolfgang Stuerzlinger, Xing-Dong Yang, and Frank Maurer. 2016. User Elicitation on Single-hand Microgestures. In Proceedings of the Conference on Human Factors in Computing Systems (CHI'16), 3403-3414.

[8] Sabrina Connell, Pei-Yi Kuo, Liu Liu, and Anne Marie Piper. 2013. A Wizard-ofOz Elicitation Study Examining Child-Defined Gestures with a Whole-body Interface. In Proceedings of the Conference on Interaction Design and Children (IDC'12), 277-280.

[9] Thomas Crespel, Patrick Reuter, and Xavier Granier. 2017. A Low-Cost Multitouch Spherical Display: Hardware and Software Design. SID Symposium Digest of Technical Papers 48, 1: 619-622.

[10] Kate Haley Goldman, Cheryl Kessler, and Elizabeth Danter. 2010. Science On a Sphere ${ }^{\circledR}$. Retrieved December 31, 2018 from https://sos.noaa.gov/What is SOS/

[11] Saul Greenberg, Sheelagh Carpendale, Nicolai Marquardt, and Bill Buxton. 2011. Sketching User Experiences: The Workbook. Elsevier.

[12] Kilem L Gwet. 2014. Handbook of Inter-Rater Reliability: the Definitive Guide to Measuring the Extent of Agreement among Raters. Advanced Analytics.

[13] Sherry Hsi and Michael Eisenberg. 2012. Math on a Sphere: Using Public Displays to Support Children's Creativity and Computational Thinking on 3D Surfaces. In Proceedings of the Conference on Interaction Design and Children (IDC '12), 248-251.

[14] Ihab Al Kabary, Marcel Büchler, and Heiko Schuldt. 2013. TOUCHify: Bringing Pen-based Touch Screen Functionality to Flat Panel Display Screens. In International Conference on Information Society (i-Society 2013), 111-116.

[15] J. Richard Landis and Gary G. Koch. 1977. The Measurement of Observer Agreement for Categorical Data. Biometrics: 159-174.

[16] Meredith Ringel Morris, Jacob O. Wobbrock, and Andrew D. Wilson. 2010. Understanding Users' Preferences for Surface Gestures. In Proceedings of Graphics Interface (GI'10), 276-268.

[17] Meredith Ringel Morris, Andreea Danielescu, Steven Drucker, Danyel Fisher, Bongshin Lee, and Jacob O. Wobbrock. 2014. Reducing Legacy Bias in Gesture Elicitation Studies. Interactions 21, 40-45.
[18] Madeline Pelz, Amanda Yung, and Celeste Kidd. 2015. Quantifying Curiosity and Exploratory Play on Touchscreen Tablets. In Proceedings of the IDC Workshop on Digital Assessment and Promotion of Children's Curiosity, 4 pages.

[19] Piaget. 1953. The Origins of Intelligence in Children. New York: Internationa Universities Press.

[20] Karen Rust, Meethu Malu, Lisa Anthony, and Leah Findlater. 2014 Understanding Child-Defined Gestures and Children's Mental Models for Touchscreen Tabletop Interaction. In Proceedings of the Conference on Interaction Design and Children (IDC'14), 201-204.

[21] Laura E. Schulz and Elizabeth Baraff Bonawitz. 2007. Serious Fun: Preschoolers Engage in More Exploratory Play When Evidence Is Confounded. Developmental Psychology 43, 4: 1045-1050.

[22] Teddy Seyed, Chris Burns, Mario Costa Sousa, Frank Maurer, and Anthony Tang. 2012. Eliciting Usable Gestures for Multi-display Environments. In Proceedings of the Conference on Interactive Tabletops and Surfaces (ITS'12), 4150.

[23] Radu-Daniel Vatavu and Ionut-Alexandru Zaiti. 2014. Leap Gestures for TV: Insights from an Elicitation Study. In Proceedings of the Conference on Interactive Experiences for TV and Online Video, 131-138.

[24] Julie R. Williamson, Daniel Sundén, and Keith Hamilton. 2016. The Lay of the Land: Techniques for Displaying Discrete and Continuous Content on a Spherical Display. In Proceedings of the ACM International Symposium on Pervasive Displays (PerDis '16), 38-44.

[25] Julie R. Williamson, John Williamson, Daniel Sundén, and Jay Bradley. 2015. Multi-Player Gaming on Spherical Displays. In Proceedings of the Conference Extended Abstracts on Human Factors in Computing Systems (CHI EA'15), 355358.

[26] Markus L. Wittorf and Mikkel R. Jakobsen. 2016. Eliciting Mid-Air Gestures for Wall-Display Interaction. In Proceedings of the Nordic Conference on HumanComputer Interaction (NordiCHI '16), Article No. 3.

[27] Jacob O. Wobbrock, Htet Htet Aung, Brandon Rothrock, and Brad A. Myers 2005. Maximizing the Guessability of Symbolic Input. In Extended Abstracts on Human Factors in Computing Systems (CHI EA' 05), 1869-1872.

[28] Jacob O. Wobbrock, Meredith Ringel Morris, and Andrew D. Wilson. 2009 User-defined Gestures for Surface Computing. In Proceedings of the Conference on Human Factors in Computing Systems (CHI'09), 1083-1092.

[29] Global Imagination: Customers. Retrieved December 31, 2018 from http://solutions.globalimagination.com/customers.html

[30] Science On a Sphere Users Collaborative Network | NOAA. Retrieved December 31, 2018 from https://www.noaa.gov/sosnetwork

[31] Pufferfish. Retrieved December 31, 2018 from https://pufferfishdisplays.com/

[32] Camera makes Puffersphere interactive - Vision Systems Design. Retrieved January 27, 2019 from https://www.vision-systems.com/ 